ISAS/EP/95/101

August 1995

\title{
Random Walk with a Boundary Line as a Free Massive Boson with a Defect Line
}

\author{
A. Valleriani 1 \\ International School for Advanced Studies \\ and \\ Istituto Nazionale di Fisica Nucleare \\ 34013 Trieste, Italy
}

\begin{abstract}
We show that the problem of Random Walk with boundary attractive potential may be mapped onto the free massive bosonic Quantum Field Theory with a line of defect. This mapping permits to recover the statistical properties of the Random Walks by using boundary $S$-matrix and Form Factor techniques.
\end{abstract}

\footnotetext{
${ }^{1}$ E-mail: angelo@frodo.sissa.it
} 


\section{Introduction}

The problem of the Random Walk in presence of a boundary line near the so-called compensation point for long chains has been solved years ago by using standard statistical mechanics methods (see [1] and references therein). It is nevertheless worth to reconsider this model in the light of recent developments in boundary Quantum Field Theory [2], in order to understand in a deeper way the connection between the classical configurations of chains and Green's functions in the corresponding Quantum Field Theory model. In this paper, we will show that the statistical problem of the 2-d Random Walk with a boundary line can be mapped onto a bosonic Quantum Field Theory with a defect line. Namely, we will see that in order to derive the statistical behaviour of the Random Walk in the presence of a boundary condition, one has to treat the boundary not as a pure classical object but as a quantum defect line in the corresponding free massive boson model, where both Reflection and Transmission amplitudes are needed. As a by-product of our results, we show that the sum of the aforementioned amplitudes plays the role of the boundary $S$-matrix for the free massive bosonic Quantum Field Theory in half-plane, such that a definition of a boundary state for this problem can be used to compute the quantities we are interested in.

The Quantum Field Theory approach presented in this paper may be useful to analyse the analogous problem with the Random Walk substituted by the Self Avoiding Walk. We would like to remind that in the bulk, many geometrical quantities of the Self Avoiding Walk can be obtained by using an $S$-matrix approach [3, 4], relying on the relationship between Self Avoiding Walks and the $O(n)$ model for $n \rightarrow 0$ [5]. This relationship has already been used to discuss several interesting aspects in the presence of a boundary condition凹.

\footnotetext{
${ }^{1}$ Fendley and Saleur $[6]$ have recently conjectured the exact boundary $S$-matrix for the Self Avoiding Walk, by using an analogy with the corresponding amplitude of the Kondo problem. It would be interesting to have a direct derivation of this quantity as a solution of the functional equations satisfied by the boundary $S$-matrix.
} 


\section{The Random Walk with boundary}

In this section, we will review some results of the Random Walk with boundary in order to establish the correspondence with the language of Quantum Field Theory. We closely follow the formulation given in ref. [7] (for the problem in the bulk, see [8] and [9]).

Let us initially consider the simplest model: the one-dimensional Random Walk on the lattice, with the walker confined to move only on the positive half-line $x \geq 0$. With a potential

$$
V= \begin{cases}\epsilon & \text { if } x=0 \\ 0 & \text { if } x \geq 1\end{cases}
$$

the partition function for the configurations is given by

$$
Z_{V}\left(x, x_{0} ; N\right)=\sum_{n_{0}=0}^{\infty} a^{n_{0}} Z\left(x, x_{0} ; N ; n_{0}\right),
$$

where $a \sim e^{-\epsilon / k T}$ and $n_{0}$ is the number of times a given path sits in the origin. The partition function $Z$ in the rhs of (2) counts the number of different configurations, in the absence of potential, of a chain of length $N$ with fixed ends $\left(x, x_{0}\right)$ and which sits $n_{0}$ times in the origin. By using the images method [10], this expression can be reduced to

$$
Z_{V}\left(x, x_{0} ; N\right)=Z_{b}\left(x, x_{0} ; N ; n_{0}=0\right)+2 \sum_{n_{0}=1}^{\infty}\left(\frac{a}{2}\right)^{n_{0}} Z\left(x, x_{0} ; N ; n_{0}\right),
$$

where $Z_{b}$ is the partition function in the bulk.

For $\epsilon<0$, there exists a critical temperature $T_{c}$ such that for $T=T_{c}$ we get $a_{c}=2$. This value of the temperature defines the so-called compensation point, where the walker does not feel any driving force, neither the (entropic) repulsion nor the (energetic) attraction. In fact, for $T>T_{c}$, we observe a preference for the walker to escape from the potential well, i.e. the favourite configurations are those which end far away from the boundary. This will be called the non-adsorbed phase of the Random Walk. On the contrary, for $T<T_{c}$ the favourite configurations are those approaching the boundary with a low probability to escape. This will be called the adsorbed phase. 
The existence of two distinct phases of the Random Walk and a critical point in between can be also established in the case of two-dimensional Random Walk with boundary [7]. In the continuum limit, in order to mimic the boundary around the compensation point, the potential can be chosen as

$$
W= \begin{cases}\infty & \text { if } x \leq 0 \\ <0 & \text { if } 0<x<b \\ 0 & \text { if } x \geq b\end{cases}
$$

and independent from the coordinate parallel to the boundary line, say $y$. Since the two-dimensional partition function of the Random Walk can be factorized into the product of two independent one-dimensional partition functions, we will study first the one-dimensional problem and then we will come back to the original two-dimensional case.

The Green's function of the Random Walk, given by the Laplace transform of the partition function $Z$, in the one-dimensional case is the solution of the differential equation

$$
\left(-\partial_{x}^{2}+m^{2}+W(x)\right) G\left(x, x_{0} ; m^{2}\right)=\delta\left(x-x_{0}\right),
$$

with the additional condition that it vanishes at infinity. The above differential equation can be solved by using standard methods (see for example [11]). Here we concentrate our attention on the solution given by

$$
G\left(x, x_{0} ; m^{2}\right)=\frac{e^{-m\left|x-x_{0}\right|}+F(m, b, T) e^{-m\left(x+x_{0}\right)}}{2 m}
$$

for $x, x_{0} \geq 0$ where all informations about the potential are encoded into the function $F$. This function can be cast into the following universal form [1] (see also [12])

$$
F=\frac{1-c / m}{1+c / m},
$$

provided that the length of the chain $\sqrt{N}>>b$ and that the function $c \propto\left(T-T_{c}\right)$ satisfies $b^{-1}<<c \leq b^{-1}$. Since we are interested in the universal behaviour of the Random Walk chains, we may let at this point

\footnotetext{
${ }^{2}$ The lower bound for $c$ negative comes from the requirement that the denominator of (7) be bigger than zero. We are supposing to have chosen a potential $W$ that satisfies these properties [7].
} 
$b \rightarrow 0$ and consider the Green's function (6) with the above function $F$ as meaningful expressions for any $x, x_{0} \geq 0$. This obviously implies that we are not concerned, from now on, with a microscopic analysis of the interaction, much like in the spirit of the $S$-matrix approach for the particle models.

We note here the following limits:

a) for $c \rightarrow+\infty$, the function $F \rightarrow-1$ and the Green's function becomes

$$
G\left(x, x_{0} ; m^{2}\right)=\frac{e^{-m\left|x-x_{0}\right|}-e^{-m\left(x+x_{0}\right)}}{2 m} .
$$

This limit corresponds to the hard-wall behaviour for $x, x_{0}$ far away from the boundary.

b) for $c \rightarrow 0$, we have instead

$$
G\left(x, x_{0} ; m^{2}\right)=\frac{e^{-m\left|x-x_{0}\right|}+e^{-m\left(x+x_{0}\right)}}{2 m} .
$$

This allows us the identification of the point $c=0$ in this description as the compensation point of the Random Walk with boundary.

Finally, it is important to notice that the Green's function (5) of the one-dimensional Random Walk can be also obtained as solution of the free differential equation in one-dimension [7]

$$
\left(-\partial_{x}^{2}+m^{2}\right) G\left(x, x_{0} ; m^{2}\right)=\delta\left(x-x_{0}\right),
$$

but with the interaction encoded into the boundary condition

$$
\partial_{x} G\left(0, x_{0} ; m^{2}\right)=c G\left(0, x_{0} ; m^{2}\right) .
$$

Once the solution of the one-dimensional case has been obtained, the Green's function of the two-dimensional Random Walk can be computed by using Fourier transform as

$$
G\left(\mathbf{r}, \mathbf{r}_{0} ; m^{2}\right)=\int_{-\infty}^{+\infty} \frac{\mathrm{d} k}{2 \pi} e^{i k\left(y-y_{0}\right)} G\left(x, x_{0} ; m^{2}+k^{2}\right)
$$

where $G\left(x, x_{0} ; m^{2}+k^{2}\right)$ satisfies the differential equation (5) and is given by (6) and (7) with the substitution $m^{2} \rightarrow m^{2}+k^{2}$. This integral can be cast 
in the suitable form

$$
\begin{aligned}
G\left(\mathbf{r}, \mathbf{r}_{0} ; m^{2}\right) & =\int_{0}^{\infty} \frac{\mathrm{d} \theta}{2 \pi} e^{i m\left(y-y_{0}\right) \sinh \theta}\left(e^{-m\left|x-x_{0}\right| \cosh \theta}+\right. \\
& \left.+\hat{S}(\theta, m, c) e^{-m\left(x+x_{0}\right) \cosh \theta}\right)
\end{aligned}
$$

where

$$
\hat{S}(\theta, m . c)=\frac{\cosh \theta-c / m}{\cosh \theta+c / m} .
$$

From the equations (8) and (9) satisfied by the one-dimensional Green's function, it is simple to derive the differential equation satisfied by the above one

$$
\left(-\Delta_{\mathbf{r}}+m^{2}\right) G\left(\mathbf{r}, \mathbf{r}_{0} ; m^{2}\right)=\delta\left(\mathbf{r}-\mathbf{r}_{0}\right)
$$

supplied with the boundary condition

$$
\left.\partial_{x} G\left(\mathbf{r}, \mathbf{r}_{0} ; m^{2}\right)\right|_{x=0}=\left.c G\left(\mathbf{r}, \mathbf{r}_{0} ; m^{2}\right)\right|_{x=0} .
$$

Notice that the above equations are those satisfied by the two-point correlation function for the euclidean massive boson with action

$$
S[\varphi]=\int \mathrm{d} x \mathrm{~d} y\left\{\theta(x)\left(\frac{1}{2}(\nabla \varphi)^{2}+\frac{m^{2}}{2} \varphi^{2}\right)+\frac{c}{2} \delta(x) \varphi^{2}\right\},
$$

where $\theta(x)$ is the Heaviside distribution.

\section{The Quantum Field Theory approach}

Aim of this section is to show that there exists a one-to-one correspondence between the problem of two-dimensional Random Walk with boundary near the compensation point and a Quantum Field Theory of a bosonic field $\varphi$ with a line of defect. In particular, we will show that the pure hard-wall situation in the Random Walk $(T \rightarrow \infty)$ is described in terms of a totally reflective defect in the Quantum Field Theory model, while the compensation point $\left(T=T_{c}\right)$ of the Random Walk corresponds to a totally transmitting defect, provided that the (classically) forbidden negative half line is mirrored to the positive axis. 
In order to establish this correspondence, the first step is to associate to each chain of the Random Walk problem a trajectory of the particle field $\varphi$ described by the Quantum Field Theory ${ }^{\prime}$. The second step consists in solving a combinatorial problem arising from the counting of the configurations. To this aim, it will be convenient to consider two copies of the Random Walk problem, defined on the left and right sides of the boundary respectively. The two copies are subjected to the same potential well and share the same temperature. In this picture, the boundary may be treated as a defect line. Notice that, since at the compensation point the behaviour of the Random Walk is like that in the absence of boundary, this corresponds, in the two-copy scheme, to trajectories that start e.g. from the right side of the boundary and end to the left side of it or viceversa. Said in other words, the compensation point is mapped into the pure transmitting behaviour of the defect line. Viceversa, the hard-wall limit of the Random Walk corresponds to purely reflecting scattering processes at the defect line.

Let us formulate more precisely this mapping. Consider the following action

$$
S\left[\varphi_{L}, \varphi_{R}\right]=S\left[\varphi_{L}\right]+S\left[\varphi_{R}\right]
$$

where

$$
S\left[\varphi_{R}\right]=\int \mathrm{d} x \mathrm{~d} y\left\{\theta(x)\left(\frac{1}{2}\left(\nabla \varphi_{R}\right)^{2}+\frac{m^{2}}{2} \varphi_{R}^{2}\right)+\frac{c}{2} \delta(x) \varphi_{R}^{2}\right\}
$$

and

$$
S\left[\varphi_{L}\right]=\int \mathrm{d} x \mathrm{~d} y\left\{\theta(-x)\left(\frac{1}{2}\left(\nabla \varphi_{L}\right)^{2}+\frac{m^{2}}{2} \varphi_{L}^{2}\right)+\frac{c}{2} \delta(x) \varphi_{L}^{2}\right\} .
$$

The fields $\varphi_{L, R}$ are not independent since are linked each other by the equation

$$
\varphi_{R}(y, x)=\varphi_{L}(y,-x) .
$$

\footnotetext{
${ }^{3}$ In the context of polymer physics, this interpretation has been proposed in [3].
} 
The equations of motion associated to the action (16) are given by

$$
\begin{gathered}
\theta(x)\left(-\nabla_{x}+m^{2}\right) \varphi_{R}=0 \\
\theta(-x)\left(-\nabla_{x}+m^{2}\right) \varphi_{L}=0 \\
\left.\partial_{x}\left(\varphi_{R}-\varphi_{L}\right)\right|_{x=0}=\left.c\left(\varphi_{R}+\varphi_{L}\right)\right|_{x=0} \\
\varphi_{R}(y, 0)=\varphi_{L}(y, 0)
\end{gathered}
$$

where the last equality comes from equation (17). Now we are in the position to see that this set of equations are the euclidean version of those solved by A.J. Bray and M.A. Moore in [13], who computed the Green's function (11), and by G. Delfino, G. Mussardo and P. Simonetti for the problem of the free relativistic massive boson with a line of defect [14]. As proved in [14], the dynamics of the massive boson with a line of defect is constrained by the integrability conditions and is completely encoded into a set of Transmission and Reflection amplitudes associated to the scattering processes of the particle hitting the defect (Figure 1). Their explicit expressions are given by [14]

$$
\begin{aligned}
T(\beta, c) & =\frac{\sinh \beta}{\sinh \beta+i c / m} \\
R(\beta, g) & =-\frac{i c / m}{\sinh \beta+i c / m}
\end{aligned}
$$

where now $\beta$ is the rapidity variable defined through the identity

$$
(E, p)=(m \cosh \beta, m \sinh \beta) .
$$

The remaining part of this letter will be devoted to the computation of the two-point correlation function of the field $\varphi$ in the presence of the defect line and to show that this quantity gives rise to the Green's function (11) of the Random Walk problem.

The correlation functions of the bosonic field $\varphi$ can be computed by using the Form Factor approach for the integrable models [15, 16]. This can be conveniently done by considering the model defined in a geometry where the boundary or the defect are placed at $t=0$. In this geometry, the boundary or defect line are promoted to quantum operators which acts on the vacuum 
of the bulk quantum theory whereas the matrix elements of the fields remain those given in the bulk case.

The Form Factors come from the insertion, in the correlation functions of a given operator $\mathcal{O}(\mathbf{r})$, of a complete set of asymptotic states

$$
\left|\theta_{1} \cdots \theta_{n}>=A^{\dagger}\left(\theta_{1}\right) \cdots A^{\dagger}\left(\theta_{n}\right)\right| 0>
$$

in such a way that for the time-ordered product of two such operators we have

$$
\begin{aligned}
& <0\left|\mathcal{O}\left(\mathbf{r}_{2}\right) \mathcal{O}\left(\mathbf{r}_{1}\right)\right| 0>=\sum_{n=0}^{\infty} \frac{1}{n !} \int \frac{\mathrm{d} \theta_{1} \cdots \mathrm{d} \theta_{n}}{(2 \pi)^{n}}\left|F_{\mathcal{O}}\left(\theta_{1}, \ldots, \theta_{n}\right)\right|^{2} . \\
& \cdot \exp \left[i m\left(y_{2}-y_{1}\right) \sum_{i=0}^{n} \sinh \theta_{i}-i m\left|t_{2}-t_{1}\right| \sum_{i=0}^{n} \cosh \theta_{i}\right] .
\end{aligned}
$$

The Form Factors are defined by

$$
F_{\mathcal{O}}\left(\theta_{1}, \ldots, \theta_{n}\right)=<0|\mathcal{O}(0)| \theta_{1} \cdots \theta_{n}>
$$

In our case, the creation and annihilation operators of the particle states satisfy the usual bosonic commutation relations

$$
\left[A(\theta), A^{\dagger}(\beta)\right]=2 \pi \delta(\theta-\beta)
$$

and this drastically simplifies the calculation of the Form Factors. In fact, for the fields $\varphi_{L, R}$ we have

$$
<0|\varphi(0)| \theta_{1} \cdots \theta_{n}>=\frac{1}{\sqrt{2}} \delta_{n, 1}
$$

while all the other non-vanishing Form Factors can be computed by using Wick's theorem based on the algebra of the operators $A(\theta)$ and $A^{\dagger}(\theta)$. With the above matrix elements, the euclidean correlation function in the bulk is given by

$$
<0\left|\mathcal{T}\left[\varphi(y, t) \varphi\left(y_{0}, t_{0}\right)\right]\right| 0>_{E}=\int_{0}^{\infty} \frac{\mathrm{d} \theta}{2 \pi} e^{-m\left|x-x_{0}\right| \cosh \theta+i m\left(y-y_{0}\right) \sinh \theta},
$$

where in the rhs we have set $i t=x$. Let us now consider the problem of computing correlation functions in the presence of the defect line. By 
considering the scattering processes as occur at the defect line in their crossed channels (Figure 2.a, 2.b), we need the new amplitudes given by

$$
\begin{aligned}
& \hat{T}(\theta, c)=T\left(i \frac{\pi}{2}-\theta, c\right)=\frac{\cosh \theta}{\cosh \theta+c / m} \\
& \hat{R}(\theta, c)=R\left(i \frac{\pi}{2}-\theta, c\right)=\frac{-c / m}{\cosh \theta+c / m} .
\end{aligned}
$$

The computation of the correlation functions in presence of the defect operator $\mathcal{D}$ can be performed by using the equations

$$
<\varphi\left(y_{1}, t_{1}\right) \cdots \varphi\left(y_{n}, t_{n}\right)>=\frac{<0\left|\mathcal{T}\left[\varphi\left(y_{1}, t_{1}\right) \cdots \mathcal{D} \cdots \varphi\left(y_{n}, t_{n}\right)\right]\right| 0>}{<0|\mathcal{D}| 0>}
$$

where the matrix elements of the operator $\mathcal{D}$ are given by [14]

$$
\begin{gathered}
<\beta|\mathcal{D}| \theta>=2 \pi \hat{T}(\beta, c) \delta(\beta-\theta) \\
<\beta_{1}, \beta_{2}|\mathcal{D}| 0>=2 \pi \hat{R}(\beta, c) \delta\left(\beta_{1}+\beta_{2}\right)
\end{gathered}
$$

and $<0|\mathcal{D}| 0>=1$.

There are two cases to consider: the first case is when the two operators $\varphi$ are across the defect line and the second one is when both fields are located on the same side with respect the defect line. With the Wick rotation $i t=x$ and the defect line placed at $x=0$, in the first case we have

$$
\begin{gathered}
<0\left|\varphi(y, x) \mathcal{D} \varphi\left(y_{0},-x_{0}\right)\right| 0>_{E}= \\
=\int_{0}^{\infty} \frac{\mathrm{d} \theta}{2 \pi} e^{-m\left(x+x_{0}\right) \cosh \theta} e^{i m\left(y-y_{0}\right) \sinh \theta} \hat{T}(\theta, c),
\end{gathered}
$$

whereas in the second case

$$
\begin{gathered}
<0\left|\varphi(y, x) \varphi\left(y_{0}, x_{0}\right) \mathcal{D}\right| 0>_{E}=\int_{0}^{\infty} \frac{\mathrm{d} \theta}{2 \pi}\left(e^{-m\left|x-x_{0}\right| \cosh \theta} e^{i m\left(y-y_{0}\right) \sinh \theta}+\right. \\
\left.+e^{-m\left(x+x_{0}\right) \cosh \theta} e^{i m\left(y-y_{0}\right) \sinh \theta} \hat{R}(\theta, c)\right) .
\end{gathered}
$$


The sum of the two contributions

$$
\begin{gathered}
G\left(\mathbf{r}, \mathbf{r}_{0} ; m^{2}\right)=<0\left|\varphi(y, x) \mathcal{D} \varphi\left(y_{0},-x_{0}\right)\right| 0>_{E}+ \\
+<0\left|\varphi(y, x) \varphi\left(y_{0}, x_{0}\right) \mathcal{D}\right| 0>_{E}
\end{gathered}
$$

is exactly the required Green's function (11).

Notice that there is another way to compute the same quantity: in fact, one could mirror the left half plane $a b$ initio to the right one and consider the Transmission amplitude as if it was a sort of Reflection amplitude. One can use this observation in order to define the function

$$
K(\theta)=\hat{R}(\theta)+\hat{T}(\theta)
$$

which together with its crossed counter part defined as

$$
K(\theta)=R\left(\frac{i \pi}{2}-\theta\right)
$$

satisfy all the conditions (boundary Yang-Baxter, boundary unitarity and boundary cross-unitarity) a boundary $S$-matrix should fulfill [2].

It is thus possible to define the boundary state at the euclidean time $x=0$

$$
\left|B>=\exp \left[\frac{1}{2} \int_{-\infty}^{+\infty} \frac{\mathrm{d} \theta}{2 \pi} K(\theta) A^{\dagger}(-\theta) A^{\dagger}(\theta)\right]\right| 0>
$$

by which we may rewrite our Green's function as

$$
G\left(\mathbf{r}, \mathbf{r}_{0} ; m^{2}\right)=<0\left|\varphi(y, x) \varphi\left(y_{0}, x_{0}\right)\right| B>_{E} .
$$

\section{Concluding remarks}

Although the original problem of the Random Walk near the compensation point is defined in half-space, it has been convenient to formulate the dynamics in terms of two copies defined for $x>0$ and $x<0$ with appropriate boundary conditions. In particular, it has been possible to identify the Transmission amplitude of the defect line model with the compensative role of the potential in the Random Walk problem and the Reflection amplitude of the 
defect line with the hard-wall limit. For a generic temperature, the dynamics is ruled by an overlapping of the two contributions, as shown in equation (25).

Note that the Quantum Field Theory with action (15) could have been solved directly by using the equations for the boundary $S$-matrix found by Ghoshal and Zamolodchikov [2]. Indeed, once the expansion of the field $\varphi$ for $x \geq 0$

$$
\varphi(x, t)=\frac{1}{\sqrt{2}} \int \frac{\mathrm{d} \theta}{2 \pi}\left[A(\theta) e^{-i m(t \cosh \theta-x \sinh \theta)}+A^{\dagger}(\theta) e^{i m(t \cosh \theta-x \sinh \theta)}\right]
$$

is inserted into the boundary condition

$$
\left.\partial_{x} \varphi\right|_{x=0}=\left.c \varphi\right|_{x=0}
$$

we have the equation

$$
\mathcal{B} A^{\dagger}(-\theta)=R(\theta) \mathcal{B} A^{\dagger}(\theta)
$$

with

$$
R(\theta)=\frac{\sinh \theta-i c / m}{\sinh \theta+i c / m}
$$

and $\mathcal{B}$ the boundary operator. The Reflection amplitude $R(\theta)$ can be used to define the boundary state (28) and the Green's function (29). However, by using this approach, the different role played by the Transmission and Reflection amplitudes of the quantum defect line would have been missed.

As our last remark, it is worth to mention that the action (15) has been extensively studied in the context of phase transitions near surfaces in [13, 17], where it describes the high-temperature Landau-Ginzburg lagrangian for a magnetic system with boundary. Its validity is restricted by the occurrence of a surface phase transition for $c<0$ : high-temperature then means temperature higher than the surface critical temperature, which is $\tau_{s}=0$ (i.e. $T_{s}=T_{c}$ ) if $c>0$ and $\tau_{s}=|c|^{2}$ if $c$ is negative. Notice that these limits are those mentioned after equation (7) and were also discussed in the context of boundary (or defect) scattering amplitudes in [14]. In the last context, the poles of $K(\theta)$ for $\tau \leq \tau_{s}$ simply imply spontaneous emission of pairs of particles from the boundary, a condition that destroys the stability of the system. 


\section{Acknowledgments}

The author would like to thank G. Mussardo for the many useful discussions and helps.

\section{References}

[1] E. Eisenriegler, K. Kremer and K. Binder, J. Chem. Phys. 77, (1982), 6269

[2] S. Ghoshal and A. Zamolodchikov, Int. J. Mod. Phys. A9, 21 (1994) 3841

[3] A. B. Zamolodchikov, Mod. Phys. Lett., A6, 19 (1991) 1807-1813

[4] J. Cardy and G. Mussardo, Nucl. Phys. B410 [FS] (1993) 451

[5] P.G. de Gennes, Phys. Lett., 38A (1972) 339

[6] P. Fendley and H.Saleur, J. Phys. A (1994) L789

[7] E. Eisenriegler, Polymers Near Surfaces (World Scientific Publishing, 1993)

[8] C. Itzykson and J.M. Drouffe, Statistical Field Theory (Cambridge University Press, 1989)

[9] E.W. Montroll and B.J. West, On an Enriched Collection of Stochastic Processes in Fluctuation Phenomena eds. E.W. Montroll and J.L. Lebowitz (Elsevier Science Publischers B.V.,1979)

[10] S. Chandrasekhar, Rev. Mod. Phys., 15 (1943) 1

[11] V.I. Smirnov, A Course of Higher Mathematics volume IV, (Pergamon Press, 1964)

[12] P.G. de Gennes, Rep. Prog. Phys. 32 (1969) 187

[13] A.J. Bray and M.A. Moore, J. Phys. A10 (1977) 1927 
[14] G. Delfino, G. Mussardo and P. Simonetti, Nucl. Phys. B432 [FS] (1994) 518

[15] F.A. Smirnov, Form Factors in Completely Integrable Models of Quantum Field Theory (World Scientific Publishing, 1992)

[16] B. Berg, M. Karowski and P. Weisz, Phys. Rev. D19 (1979) 2477;

M. Karowski and P. Weisz, Nucl. Phys. B139 (1978) 455

[17] D.L. Mills, Phys. Rev. B3, 11 (1971) 3887;

K. Binder and P.C. Hohenberg, Phys. Rev. B6, 9 (1972) 3461;

T.C. Lubensky and M.H. Rubin, Phys. Rev. B12 (1975) 3885;

K. Binder, in Phase Transitions and Critical Phenomena, Vol. 8 (C. Domb and J.L. Lebowitz,eds), Academic Press, London (1983) p. 1 


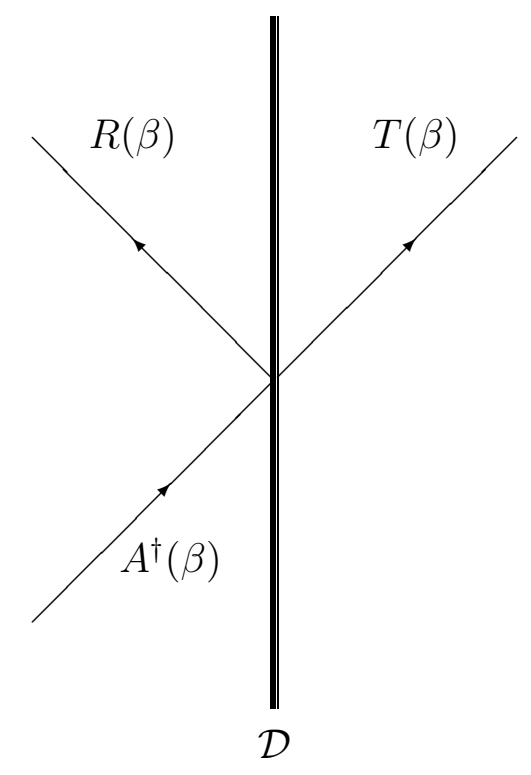

Figure 1

The particle hits the defect line and is scattered according to the Reflection and Transmission amplitudes. 


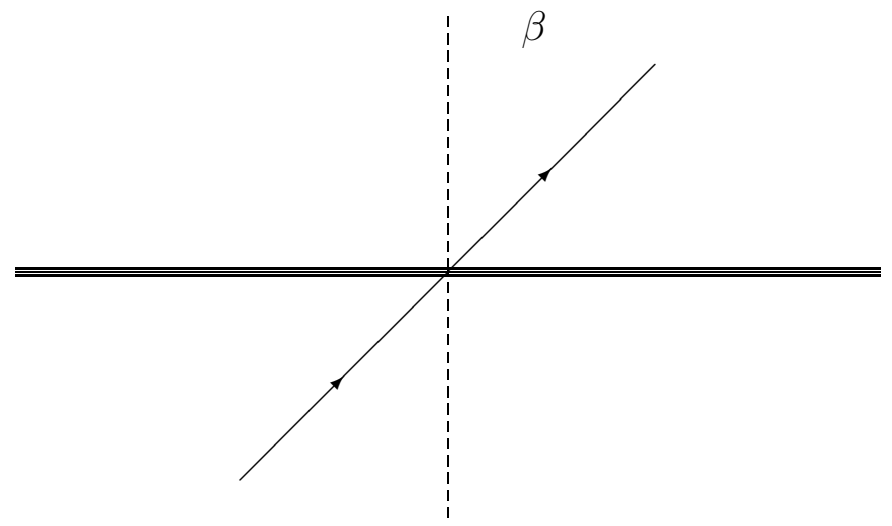

Figure 2.a

The process of transmission

with the defect line at $i t=x=0$. 


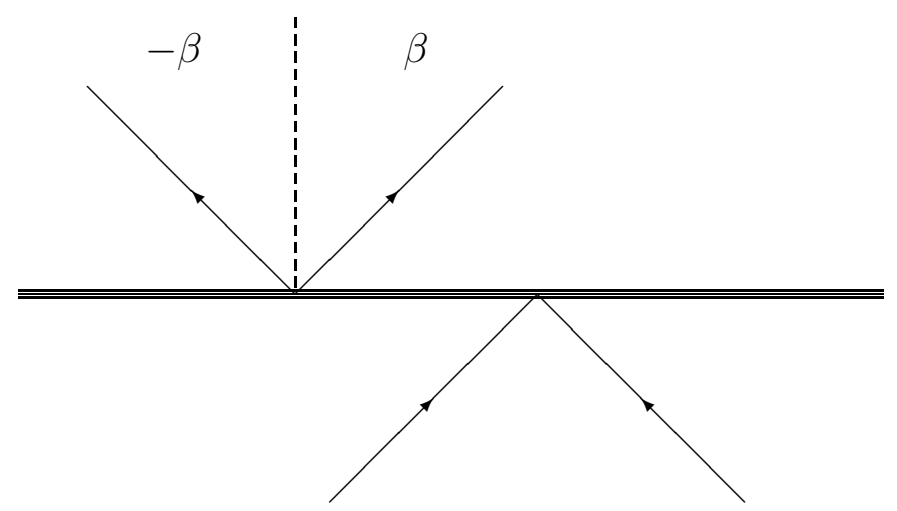

Figure 2.b

The process of reflection with the defect line at $i t=x=0$. 